\title{
An Energy Efficient Virtual Machine Migration Method in Cloud
}

\author{
Hongtao Liang ${ }^{1,2, a}$, Jianliang $\mathrm{Xu}^{1, \mathrm{~b}}$, Min Yuan ${ }^{3, \mathrm{c}}$, Mingtao Liu ${ }^{1, \mathrm{~d}}$, \\ Xiaohong Wang ${ }^{1, \mathrm{e}}$ \\ ${ }^{1}$ Collage of Information Science and Engineering, Ocean University of China, \\ Qingdao 266100, China \\ ${ }^{2}$ Qingdao University of Technology, Qingdao 266300, China \\ ${ }^{3}$ Xi'an Institute of Prospecting and Mapping, Xi'an 710054, China \\ aemail: hongtaoliang@126.com, bemail: xjl9898@ouc.edu.cn, 'email: \\ yuanmin2020@126.com, demail: liumingta0412@hotmail.com, eemail:wxh_qhnu@163.com
}

\section{Keywords: Energy Efficient; Virtual Machine; Migration Method; Cloud Computing}

\begin{abstract}
Cloud computing, as a newly emerged service oriented paradigm, is emerging as a leading solution for on-demand services. One of the challenges faced by current cloud is to decide how to migrate virtual machine to another physical machine. This paper presents an energy efficient virtual machine migration method in cloud. Power consumption of physical machine and network overhead are taken into account. The power consumption curve for virtual machine migrating process is illustrated. The experiments verify the effectiveness of our proposed method with low overhead.
\end{abstract}

\section{Introduction}

In recent years, cloud computing, as an emerging technology gaining more attentions both in IT industry and academic research is rapidly growing [1,2]. Cloud computing, like all other four existing service model, will provide the basic level of computing service that is considered essential to meet the increasing everyday computation and storage needs. Cloud computing offering utility-oriented IT services to client has started hosting a wide range of various client applications. Based on a pay-as-you-go service model, cloud computing is considered as one of the evolving paradigms that enable providing computing resources as services [3].

CSPs (Cloud Service Providers) provide its clients massively scalable resources like CPU processing, storage, software application. Massive infrastructures are managed by virtualization technologies in cloud computing, working as a virtual infrastructure resource pool $[4,5]$. This virtual infrastructure resource pool is configured or reconfigured by centralized entity which is responsible for virtual machine migration among physical machine [6, 7]. Our research hypothesis is that a more conservation method can be used to guarantee steadier performance. Different from the existing methods for virtual machine migration, our method focuses on energy efficient.

\section{System Overview}

The Fig. 1 shows the system architecture of our proposed system. In general, there are 3 main components in system: (1) Scheduler is used to migrate virtual machine, (2) PMM (Performance Monitor Module) is used to monitor the current load of every virtual machine, (3) NMM (Network Monitor Module) is designed to monitor the traffic between physical machine, and scheduler is used to execute the live migration of virtual machine among physical machines in cloud based on the analysis results by PMM and NMM functional module. Cloud computing builds heavily on capabilities available through virtualization technology.

In theory, virtualization technology means that multiple virtual machines can share a single physical machine at the same time. This process, known as server consolidation, is used by cloud center to increase resource utilization and reduce power consumption costs. In our framework, we 
take full advantage of virtualization technology. PMM and NMM modules connected every virtual machine sharing a physical machine collect necessary running time parameters used to analysis.

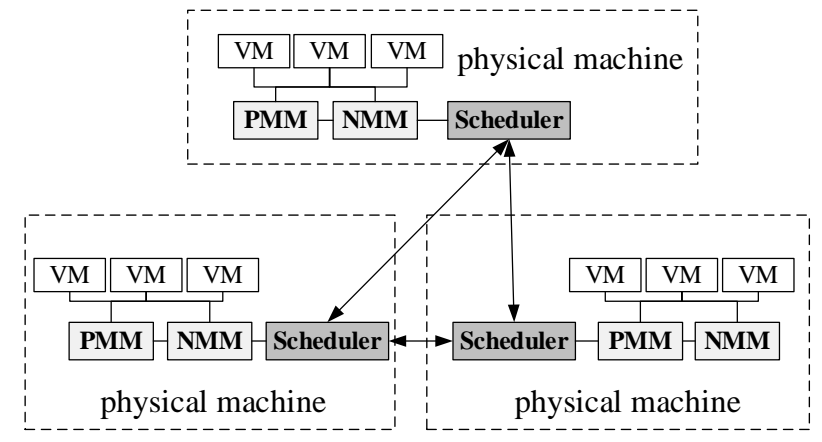

Fig.1 System architecture

\section{The proposed Strategy}

In this section, we will give our proposed strategy in detail. Suppose $p=\{p 1, p 2, \ldots, p n\}$ denote the physical machine in cloud, $n$ is the number of physical machine, and $\mathrm{pi}(1 \leq \mathrm{i} \leq \mathrm{n})$ stands for i physical machine. Given task is running in virtual machine. PMM can monitor and record the CPU frequency in real-time. Let fi denote the CPU frequency on pi. Hi is the power consumption on pi. We apply the power consumption model introduced by Zhu et al.[8], which can be summarized as formulation (1).

$H_{i}=c_{e} f_{i}^{m}+b_{i}$

In formulation (1), where $c_{e} \cdot f_{i}^{m}$ is frequency-dependent power, in which $c_{e}$ is the effective switching capacitance and $m$ is the dynamic power exponent. $b_{i}$ is the sum of the sleep power consumption which is used to maintain basic circuits and the frequency-independent power.

Let $v c_{i}$ denote the size of virtual machine image $i$. The virtual machine image should be compressed before it be migrated to another physical machine in order to save network bandwidth. Suppose $k$ is compression ratio by a given compression algorithm. Hence the time needed to finished compression is given by formulation (2).

$t=\frac{\alpha \cdot v c_{i}}{k \cdot f}$

Specifically, $\alpha$ is a platform-dependent constant in above formulation (2). Obviously, the power consumption $p c_{i}$ which is used to compress the virtual machine image is closely related to $H_{i}$ and $t$. So, $p c_{i}$ can be given by following formulation (3).

$$
p c_{i}=H_{i} \cdot t_{i}=\left(c_{e} f_{i}^{m}+b_{i}\right) \frac{\alpha \cdot v c_{i}}{k \cdot f}
$$

Based on formulation (2), the virtual machine image is compressed by a certain compression ratio $k$. And the size of compressed image $v c_{i}$ can be given by formulation (4).

$v c_{i}=(1-k) \cdot b n$

Suppose $n b_{i}$ denote the network bandwidth of virtual machine $i$. The time migrated virtual machine $i$ is $t_{n}$ by network. Obviously, $t_{n}$ is given by formulation (5).

$$
t_{n}=\frac{v c_{i}}{n b_{i}}
$$

Based on the above analysis, the total power consumption used to migrate a virtual machine image contains two main parts. The first is the compressing process and the second is network transferring process. Let $y$ denote the total power consumption for the whole migrating process. Consequently, $y$ can be given by formulation (6).

$y=p c_{i}+t_{n} \cdot H_{i}$ 
In order to meet the goal of energy efficient, we need to try to find the optimal compressiong ratio $k_{\text {opt }}$. And, apparently, $k_{\text {opt }}$ can be obtained by following formulation (7).

$k_{\text {opt }}: \frac{d g_{Y}(k)}{d k}=0$

From above discussion, the virtual machine cloud be migrated to other physical machine by optimal compression ratio $k_{\text {opt }}$, which not only saves physical machine power consumption but it also saves network bandwidth.

\section{Experimental Analysis}

To evaluate the validity of our proposed strategy, a site of real computation-intensive application HPL is running in simulated cloud platform in following experiment. Openstack has been chosen as the platform for simulating as cloud, which is contains 64 same virtual machine sharing 4 same HP DL338 servers (Intel Xeon E5 CPU, 8G DDR4 RAM). Available network bandwidth among physical machines is $1000 \mathrm{Mbps}$.

Firstly, we evaluate the overhead of our proposed strategy. We launched 4 to 64 virtual machines and recode the overhead by formulation (7) to get the optimal compression ratio $k_{\text {opt }}$. Those experimental results are shown as in Fig. 2 in detail.

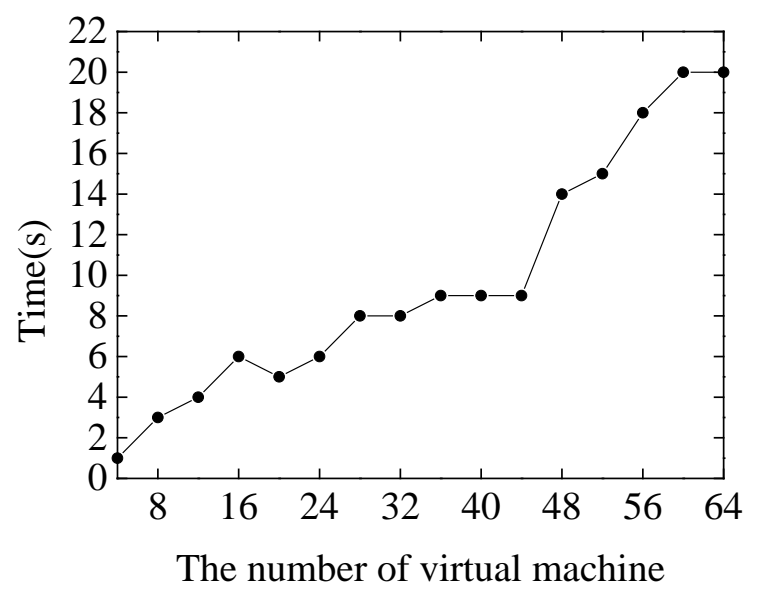

Fig. 2. Overhead of our proposed strategy

From Fig. 2, we can see that the overhead is increasing with the number of virtual machine increased, but this increasing tendency is approximately a linear model besides the input parameters and variables used in experiment settings. This is because, our strategy does not introduce considerable computational complexity. That is to say, our strategy performs extremely very well for getting the optimal compression ratio $k_{\text {opt }}$. And the overhead of our proposed strategy is acceptable in practice.

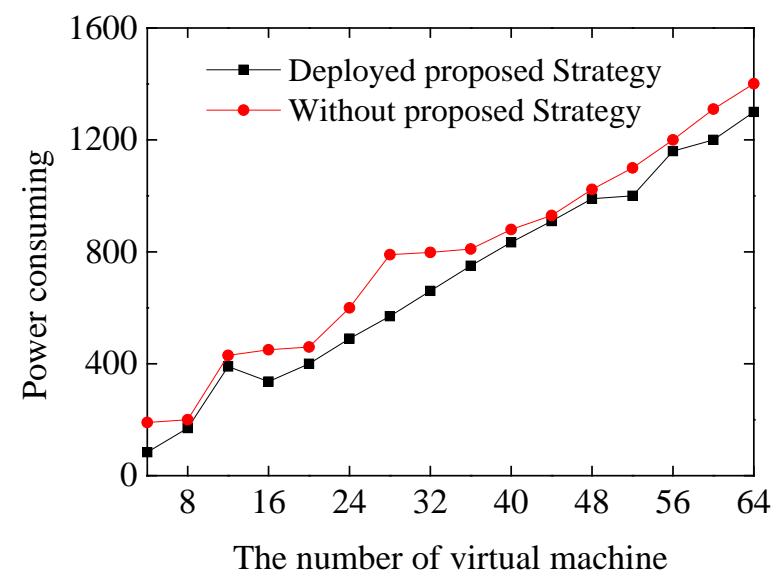

Fig. 3. The effectiveness of our proposed strategy 
Then, we evaluate the effectiveness of our strategy. As illustrated above, one of the main objective of our framework is energy efficient. We migrate virtual machines to physical machine in cloud by random migration method and by our proposed migration strategy. Fig. 3 depicts those experiment results in detail.

From Fig.3, we can conduct that our proposed strategy always consumes smaller power than without deploying out strategy. In order to get minimal the power consumption for virtual machine migration, our strategy compresses the virtual machine image by optimal compression ratio before migrating. Meanwhile, the strategy can also save network bandwidth. It is important to note that our proposed method is an energy efficient way.

\section{Conclusion}

Total power consumption is a key parameter for virtual machine migration in cloud computing. In this paper, we present how to migrate the virtual machine in cloud in an energy efficient way. The optimal compression ratio and network bandwidth are considered in this method. From the observed simulation results, the experiment results demonstrate effectiveness of our proposed method with low overhead.

\section{Acknowledgements}

In this paper, the research was sponsored by the Science and Technology Project of Shandong Province University (Project No. J13LN77) and Shandong Province Colleges and Universities Young Teachers Visiting Scholar Funded.

\section{References}

[1] R. W. Ahmad, A. Gani, S. H. Hamid, M. Shiraz, A. Yousafzai, F. Xiab: A Survey on Virtual Machine Migration and Server Consolidation Frameworks for Cloud Data Centers [J]. Journal of Network and Computer Applications, 2015: 11-25.

[2] M. R. Desai, H. B. Patel: Efficient Virtual Machine Migration in Cloud Computing[C]. 2015 Fifth International Conference on Communication Systems and Network Technologies (CSNT), 2015: 1015-1019.

[3] M. Melo, P. Maciel, J. Araujo, R. Matos, C. Araujo: Availability Study on Cloud Computing Environments: Live Migration as a Rejuvenation Mechanism[C]. 2013 43rd Annual IEEE/IFIP International Conference on Dependable Systems and Networks (DSN), 2013: 1-6.

[4] A. Jaikar, D. Huang, G. Kim, S. Noh: Power Efficient Virtual Machine Migration in a Scientific Federated Cloud[J]. Cluster Computer, 2015, 18(2): 609-619.

[5] T. Yapicioglu, S. Oktug: A Traffic-aware Virtual Machine Placement Method for Cloud Data Centers[C]. 2013 IEEE/ACM 6th International Conference on Utility and Cloud Computing, 2013: 299-301.

[6] C. Kim, C. Jeon, W. Lee, S. Yang: A Parallel Migration Scheme for Fast Virtual Machine Relocation on a Cloud Cluster[J]. Journal of Supercomputing, 2015: 4623-4645.

[7] S. Jamali, S. Malektaji: Improving Grouping Genetic Algorithm for Virtual Machine Placement in Cloud Data Centers[C]. 4th International Conference on Computer and Knowledge Engineering, 2013: 328-333.

[8] D. Zhu, R. Melhem, D. Mosse, E. Elnozahy: Analysis of an Energy Efficient Optimistic TMR Scheme[C]. 10th International Conference on Parallel and Distributed Systems, 2004: 559-568. 